\title{
Care und Case Management \\ - Steuerung im Kontext von Pflegebedürftigkeit
}

Thomas Klie

11.1 Einleitung - 166

11.2 Case Management und Langzeitpflege: Im Mittelpunkt die Person? - 167

11.3 Case Management lege artis - 168

11.4 Case Management und Empirie - 170

11.5 Steuerungsfunktionen des Care und Case

Managements - 172

11.6 Ausblick - 174

Literatur - 175 


\section{- Zusammenfassung}

Mit dem Pflege-Weiterentwicklungsgesetz 2008 hat der Gesetzgeber Care und Case Management-Strategien sowohl in das Leistungsrecht als auch in die Strukturvorgaben der Pflegeversicherung aufgenommen. Angesichts der Unübersichtlichkeit der Hilfestrukturen, insbesondere im Zusammenhang mit Schnittstellen zwischen den Sektoren oder auf der Fallebene, wurden ein Rechtsanspruch auf Pflegeberatung gemäß $\& 7 a$ SGB XI im Sinne von Case Management eingeräumt und für Vernetzungs- und Koordinationsstrukturen im Sinne des Care Managements bundesgesetzliche Rahmenbedingungen und Aufgabenbeschreibungen formuliert. Case Management-Arbeitsansätze können in der Lage sein, die in der deutschen Pflegeversicherung eher vernachlässigte Personenzentrierung stärker zu betonen. Ein Care und Case Management lege artis verschränkt systematisch die Fall- mit der Systemebene. Dass dies in der Praxis in Deutschland fast nirgendwo konsequent umgesetzt wird, arbeitet der Beitrag ebenso heraus wie die Steuerungsfunktionen des Care und Case Managements, die verbunden werden müssen mit systematischen Monitoring-Instrumenten und Planungsprozessen auf regionaler und kommunaler Ebene. Care und Case Management-Strukturen in der Langzeitpflege sind alternativlos, aber zugleich voraussetzungsvoll.

With the Long-Term Care Further Development Act of 2008, the German legislator introduced care and case management strategies into both benefit law and the structural requirements of long-term care insurance. In view of the complexity of the support structures, particularly in connection with interfaces between sectors or at the case level, a legal right to advisory support in accordance with $₫ 7 a S G B X I$ in the sense of case management was granted and networking and coordination structures in the sense of care management were initiated. Case management approaches may allow placing greater emphasis on a person-centred approach, which is rather neglected in German long-term care insurance. Care and case management systematically links the case and system levels. The article shows that this is not consistently implemented anywhere in Germany and also highlights the control functions of care and case management, which must be combined with systematic monitoring instruments and planning processes at the regional and municipal level. There is no alternative to care and case management structures in longterm care, but at the same time quite demanding.

\subsection{Einleitung}

Wohin nach Krankenhausentlassung, was tun bei Dekompensation der von Angehörigen getragenen häuslichen Pflege, welche Alternativen zum Sterben im Krankenhaus lassen sich organisieren, wenn die Präferenz heißt: Sterben daheim? Kein ambulanter Pflegedienst verfügbar, keine Rehaklinik mit freien Betten trotz Rehabedarfs; die Indikationen für Case Management in der Langzeitpflege sind vielfältig. Und keineswegs beschränken sich die Fallkonstellationen allein auf Versorgungsfragen: Wie gelingt es, Zugang zu einem Menschen zu finden, der mit seinem Haushalt und seiner Hygiene gar nicht mehr zurechtkommt, wie lässt sich erwachsenschutzrechtlich relevanten Situationen wie freiheitsentziehenden Maßnahmen oder anderen Formen der Gewalt in der häuslichen Pflege begegnen? All diese Praxisfragen machen deutlich: Für die Steuerung der Versorgung in der Langzeitpflege kommt dem Care und Case Management sowohl auf der Fall- als auch auf der Systemebene eine in mehrfacher Hinsicht bedeutsame Rolle zu. Dies gilt für das Case Management als Verfahrensweise in Humandiensten, aber auch für Case Management als Organisation (Monzer 2018) regionaler Akteure der für die Langzeitpflege relevanten angrenzenden Sektoren wie der gesetzlichen Krankenversicherung, der Teilhabe, den Kommunen, Einrichtungen und Diensten sowie den zivilgesellschaftlichen Akteuren. 


\subsection{Case Management und Langzeitpflege: Im Mittelpunkt die Person?}

Personenzentrierung lautet seit einiger Zeit das Paradigma der Leistungsgewährung in der Eingliederungshilfe. Auf das Individuum zugeschnittene Leistungen, mit ihm ausgehandelt, seine Ressourcen reflektierend und seine Präferenzen in der Lebensgestaltung würdigend. Das gelingt mitnichten immer und überall. Gleichwohl ist, durchaus auch leistungsrechtlich hinterlegt, die Personenzentrierung - verbunden mit dem Verzicht auf die rechtliche Sonderstellung von stationären Einrichtungen - zur verbindlichen Vorgabe im Teilhaberecht avanciert. ${ }^{1}$ Auch wenn Pflegebedürftige im Rechtssinne Menschen mit Behinderungen sind, auch wenn es für Menschen mit Pflegebedarf und ihre An- und Zugehörigen immer und zuvörderst darum gehen muss, dass sie in ihrer individuellen Lebenslage gesehen und ihr Wunsch- und Wahlrecht hinsichtlich der Gestaltung der Hilfen im Kontext von Pflegebedürftigkeit gewahrt wird, hat die Personenzentrierung in der sozialen Pflegeversicherung noch keine hervorgehobene Bedeutung erlangt. Die deutsche Pflegeversicherung setzt auf die Selbstorganisation von Familien, die Solidarität von $\mathrm{An}$ - und $\mathrm{Zu}$ gehörigen und die Bereitstellung von formellen Diensten und Einrichtungen, die - orientiert am Recht der gesetzlichen Krankenversicherung - qualitätsgesicherte Leistungen in Modulform respektive in hinsichtlich ihrer Leistungen inhaltlich determinierten Einrichtungen vorhält. $\mathrm{Ob}$ und inwieweit auf diese Weise tatsächlich individuelle Bedarfskonstellationen, Lebenslagen, Lebenswelten und Präferenzen der Lebensführung berücksichtigt und realisiert werden können, steht nicht systematisch im Blick der Pflegeversicherung. Im Fokus ist die Qualitätssicherung von Einrichtungen und

\footnotetext{
Fachverbände der Behindertenhilfe (Hrsg) (2010) 10 Thesen zur Personenzentrierung. https://www. diefachverbaende.de/files/stellungnahmen/201010-01-Thesen-zur-Personzentrierung.pdf. Zugegriffen: 05. Februar 2020.
}

Diensten - incl. der Messung der Lebensqualität der Pflegebedürftigen.

Im Vorfeld der Wahl eines Pflegearrangements und den entsprechenden Entscheidungsprozess flankierend steht den auf Pflege angewiesenen Menschen und ihren Angehörigen rechtlich ein Spektrum von auf sie zugeschnittenen Beratungsangeboten $\mathrm{zu}$, die sie bei der Bewältigung von den mit der „Pflegebedürftigkeit" verbundenen An- und Herausforderungen unterstützen sollen. Dazu gehören Beratungsangebote, sei es gemäß $₫ 7$ SGB XI durch die Pflegekassen, in Pflegestützpunkten soweit sie vorhanden sind - gemäß §7c SGB XI oder durch kommunale Angebote der Altenhilfe und Seniorenberatung vor Ort. Auch über Pflegekurse gemäß $\$ 45$ SGB XI und Pflegeberatungsbesuche gemäß $\$ 37$ Abs. 3 SGB XI werden auf die Personen respektive den Haushalt zugeschnittene Beratungs- und Unterstützungsleistungen (zum Teil verpflichtend) angeboten. Beratung findet überdies im Zusammenhang mit Krankenhausentlassungen durch den dortigen Krankenhaussozialdienst und, soweit vorhanden, durch das den Krankenhäusern übertragene Versorgungsmanagement gemäß $₫ 11$ SGB V statt. Kassen sind verpflichtet, für Palliativpatienten eine gesonderte Palliativberatung gemäß $₫ 39 \mathrm{~b}$ SGB V anzubieten. Dass damit der Hilfe-, Beratungs- und Unterstützungsbedarf von auf Pflege angewiesenen Menschen und ihren An- und Zugehörigen nicht immer in befriedigender Weise gedeckt wird, zeigen etwa die Zahlen der AllensbachUmfrage im Rahmen des DAK-Pflegereports 2019, wonach die meisten auf Pflege angewiesenen Menschen sich Beratung bei Freunden, Familienangehörigen und beim Hausarzt holen (Haumann 2018). Dass es vielfach an Infrastrukturangeboten vor Ort fehlt, die sich an Versorgungswünschen orientieren, ist ebenso dokumentiert (Klie 2018): Von gleichwertigen Lebensbedingungen in der Pflege in Deutschland kann nicht die Rede sein. Das gilt in besonderer Weise für das Case Management. All die den Sachverständigenrat im Gesundheitswesen immer wieder beschäftigenden Schnittstellenprobleme in einer sektorenübergreifen- 
den Versorgung, die die Notwendigkeit von Case Management provozieren, schlagen auch und gerade bei auf Pflege angewiesenen Menschen auf. ${ }^{2}$ Krankenhausentlassungen, die Zusammenarbeit zwischen stationärem und ambulantem Sektor sind sowohl innerhalb der GKV als auch zwischen GKV und SPV trotz vielfältiger Bemühungen notleidend.

Die Zahlen über die Überforderung pflegender Angehöriger, ihren Wunsch, Pflegeaufgaben schnellstmöglich abzugeben (Rothgang und Müller 2019), aber auch die Zahlen über die Inanspruchnahme von osteuropäischen Haushaltshilfen (Petermann et al. 2017) verweisen darauf, dass aus vielfältigen, vor allem in der häuslichen Pflege liegenden, Gründen eine Weiterentwicklung und Qualifizierung der Pflegesicherung in Deutschland geboten ist.

Mit dem Pflege-Weiterentwicklungsgesetz wurde 2008 den schon damals bestehenden und bekannten Problemlagen insofern begegnet, als in Modellprojekten erprobte Care und Case Management-Strukturen in die Konzeption und in das Leistungsrecht der Pflegeversicherung aufgenommen wurden: $₫ 7$ a SGB XI, unrichtigerweise als Pflegeberatung bezeichnet, räumt dem einzelnen Versicherten einen Rechtsanspruch auf Case Management ein. ${ }^{3}$ Explizit hat der Gesetzgeber in der Begründung zu $₫ 7$ a SGB XI (Eisfeld und Krahmer in Klie et al. 2014) ein auf das Niveau von DGCCStandards verpflichtetes Case Management vor Augen gehabt, als er den Rechtsanspruch im SGB XI verankert hat. Die Care ManagementStrukturen, die durch Pflegestützpunkte bundesweit eingeführt werden sollten, konnten mangels Einigung mit den Ländern nicht flä-

Sachverständigenrat zur Begutachtung der Entwicklung im Gesundheitswesen (2018) Bedarfsgerechte Steuerung der Gesundheitsversorgung. https:// www.svr-gesundheit.de/fileadmin/user_upload/ Gutachten/2018/SVR-Gutachten_2018_WEBSEITE. pdf. Zugegriffen: 05. Februar 2020.

3 Pflegeberatung heißt in einem professionstheoretischen Sinne Beratung durch Pflegekräfte im Kontext von Pflege als Teil des Pflegeprozesses (Koch-Straube 2008). chendeckend und gleichmäßig implementiert werden. Hier hat sich eine höchst differente Versorgungslage entwickelt (Braeseke et al. 2013). Auch die Implementierung der Pflegeberatung ist keineswegs einheitlich und zufriedenstellend. Das haben die jeweiligen Evaluationen zu $₫ 7$ a SGB XI deutlich gemacht (Klie 2011, Braeseke et al. 2019).

Welche Ziele werden lege artis mit dem Case Management verbunden, was kann und soll Case Management im Kontext von Pflegebedürftigkeit leisten? Sollen die Pflegebedürftigen "gesteuert" werden? Steuert das Case Management den auf Pflege angewiesenen Menschen durch das System? Dient das Case Management dem auf Pflege angewiesenen Menschen und seinen An- und Zugehörigen als advokatorische Unterstützung in einer komplexen Lebenslage und in existenziellen Grenzsituationen? Oder steht die Steuerung im Interesse der Institutionen - etwa Krankenhäusern - unter gesundheitsökomischen Restriktionen im Vordergrund?

\subsection{Case Management lege artis}

Case Management ist nach der Definition der Deutschen Gesellschaft für Care und Case Management eine Verfahrensweise in $\mathrm{Hu}-$ mandiensten und ihrer Organisation zu dem Zweck, bedarfsentsprechend im Einzelfall eine nötige Unterstützung, Behandlung, Begleitung, Förderung und Versorgung von Menschen angemessen zu bewerkstelligen. Der Handlungsansatz ist zugleich ein Programm, nach dem Leistungsprozesse in einem System der Versorgung und in einzelnen Bereichen des Sozialund Gesundheitswesens effektiv und effizient gesteuert werden können. ${ }^{4}$ Case Management wird mit dieser Definition aus seiner Beliebigkeit herausgeholt, die im Sprachgebrauch gerade auch in der Langzeitpflege verbreitet ist. Es wird aber zugleich auch die Komplexität

4 DGCC (2013) Was ist Case Management? https:// www.dgcc.de/case-management. Zugegriffen: 05. Februar 2020. 
des Case Management-Ansatzes deutlich. Zudem wird die Zweidimensionalität von Care und Case Management sichtbar: Case Management zielt nie nur auf die Fall-, sondern immer auch auf die Ebene der Systemsteuerung. Um das Case Management weiter zu profilieren, sind folgende Aspekte und Konkretisierungen zu ergänzen: Ziel des Case Management ist die Organisation und Aufrechterhaltung von Unterstützungssystemen, die sich auf der einen Seite an den individuellen Bedürfnissen der jeweiligen Personen orientieren, im Kontext der Langzeitpflege aber auch an ihren An- und Zugehörigen. Zum anderen initiiert und organisiert das Case Management aus der Kenntnis der Einzelfälle auch fallübergreifende Versorgungsnetzwerke. Sie sollen die Zusammenarbeit unterschiedlicher Unterstützungsbereiche, benachbarter Sektoren mit ihren jeweiligen Zugangs- und Zuständigkeitsregeln erleichtern (Monzer 2018, S. 1).

Versteht man Case Management als einen Arbeitsansatz, gilt neben der systematischen Organisation der Einzelfallarbeit immer auch die angemessene Nutzung der informellen Ressourcen der Person, des Gemeinwesens als Aufgabe und Gegenstand. Gemeinsam mit institutionellen und Ressourcen formeller Angebote des Arbeitsfeldes sind sie im Sinne eines wohlfahrtspluralistischen Hilfemixes zu koordinieren (Klie 2020). Gerade in der Langzeitpflege, in der es darum geht, Pflegearrangements, die niemals nur formelle Hilfen kennen (sollten), zu gestalten, zu qualifizieren und weiterzuentwickeln, ist Case Management weit mehr als die Vermittlung formeller Hilfen - sei es ambulanter Dienste, Pflegehilfsmittel oder Maßnahmen des Wohnungsumbaus. Case Management zielt zumindest in ambulanten Settings immer auf wohlfahrtspluralistische Arrangements.

Als Perspektive gilt im Case Management eine ökosoziale und systemische Orientierung. Auf diese Weise kann vor allem in komplexen und komplizierten Problemlagen die erforderliche Unterstützung so koordiniert werden, dass durch neue Kooperationen respektive die Qualifizierung bestehender Kooperationen möglichst wenig zusätzliche Angebote geschaffen werden müssen. Durch die qualitativ sinnvolle Kombination von formellen und informellen Ressourcen gilt es Lösungen zu entwickeln, die an der jeweiligen Lebenslage, das heißt der Ressourcensituation der Betroffenen, orientiert sind.

Die Aufgabe des Case Management besteht jeweils in der Unterstützung von Personen, die unter den gegebenen persönlichen und institutionellen Voraussetzungen Probleme haben, die für sie erforderlichen Hilfen in einer Weise in Anspruch zu nehmen, die ihre Lebenslage stabilisiert, Probleme beseitigt und Gefährdungen vorbeugt. Damit fällt dem Case Management vorrangig die Funktion zu, die Unterstützung zu organisieren, die der Betroffene nicht selbst erreichen kann. Folgt man dieser Prämisse, müssen möglichst solche Hilfen organisiert werden, die zu seiner Lebenswelt im Sinne seiner kulturell geprägten Lebensweise und des ihn umgebenden Umfeldes passen und letztlich von ihm und seiner Umgebung ohne weitere Hilfestellung genutzt und/oder organisiert werden können. Damit ist Case Management in der Regel nicht für die direkte Leistungserbringung zuständig. Sie organisiert diese vielmehr.

Schließlich geht es um den Gegenstand der Leistung des Case Managements. Dies sind primär die Fälle. Die Bezeichnung „Case Management" legt nahe, dass hier nicht Menschen oder Klienten gemanagt werden. Es geht nicht um „Pflegefälle“ in der inhumanen Ausdrucksweise, die in der Bevölkerung verbreitet ist (Klie 2014). Es werden Cases, es wird also das gemanagt, was gemeinsam als „der Fall“ identifiziert und bezeichnet wurde. Selbstverständlich geht es dabei auch und gerade darum, zu den am Fall Beteiligten in vertrauensgeprägte Beziehungen einzutreten, sie zu entwickeln und zu pflegen. Case Management ersetzt nicht Beziehungen, sondern baut auf diesen auf. Im Sinne der Fallsteuerung ist das Case Management nicht für alle Rat suchenden, auf Pflege angewiesenen Menschen und ihre An- und Zugehörigen zuständig. Case Management ist für komplexe Fallkonstellationen da; nur diese indizieren Case Management. Die Problemdichte, die Vielfalt der beteiligten Akteure, die 
fehlenden Routinen in der Bearbeitung der jeweiligen Fallkonstellationen und Infrastrukturdefizite können Case Management indizieren. Dabei gehört zum Case Management als methodisch elaborierte Verfahrensweise eine bestimmte Abfolge, die von der Fallklärung (Case Finding) über das Assessment, das weit über das NBI (neues Begutachtungsinstrument) im Fall der Pflegebedürftigkeit hinausgeht, und die Hilfeplanung bis hin zur Leistungs- und Angebotssteuerung greift. Bedeutsam sind auch die unterschiedlichen Rollen des Case Managements respektive seine Funktion. Üblicherweise werden vier Funktionen unterschieden:

1. Die Advocacy-Funktion: Hier geht es um die im Einzelfall erforderlichen Ressourcen, die in ausreichendem qualitativem und quantitativem Maße erschlossen werden sollen. In der Advocacy-Funktion übernimmt das Case Management eine sozialanwaltliche Rolle und sieht sich an der Seite des Klienten zur Stärkung seiner Position. Aus der Kenntnis seiner individuellen Situation und Bedürfnislage ist es die Aufgabe des Case Managements, die Angebote und Maßnahmen durchzusetzen, die notwendig und zur Lebenswelt passend sind. Auch menschenrechtlich relevante Gefährdungssituationen sind dabei mit zu reflektieren.

2. Die Broker-Funktion knüpft an die gute Kenntnis der Angebotslandschaft von Case Managern. Hier übernimmt das Case Management eine neutrale beratende Vermittlungsfunktion hinsichtlich Diensten und Einrichtungen. Die Unübersichtlichkeit der Angebote oder unbekannte Unterstützungsoptionen werden ebenso in den Blick genommen wie die Passfähigkeit von regional vorhandenen Angeboten.

3. In der Gatekeeper-Funktion übernimmt das Case Management die Funktion, einem Fall die richtigen und notwendigen sowie vorrätigen Ressourcen zuzuteilen. Hier allerdings hat das Case Management auch die Aufgabe, Leistungen auszuschließen, für die eine Berechtigung nicht besteht.

4. In der Social-Support-Funktion schließlich geht es um die Befähigung der Klienten, sich mit notwendigen Hilfeangeboten auseinanderzusetzen und Entscheidungen zu treffen respektive auch die eigene Situation neu zu deuten und sich für neue Lösungen zu öffnen, die bisher nicht mit in Betracht gezogen wurden.

Damit sind Funktionen und das Handlungskonzept des Case Managements skizziert, das in der Langzeitpflege sowohl auf der Fall- als auch auf der Systemebene mit dem Gesetz zur strukturellen Weiterentwicklung der Pflegeversicherung (Pflege-Weiterentwicklungsgesetz) vom 28. Mai 2008 eingeführt wurde selbstverständlich nicht unter der Bezeichnung "Case Management", sondern unter den Begriffen Pflegeberatung und Pflegestützpunkte. Case Management ist sowohl eine Methode der Fallarbeit als auch ein Prozess- und Strukturmerkmal von Organisationen. Es ist ein Kooperationsmerkmal von Netzwerken und durch die Pflegeversicherung Vorgabe der Pflegepolitik geworden.

\subsection{Case Management und Empirie}

Die deutsche Situation der Pflegeberatung und der Pflegestützpunkte gleicht einem föderalen und kassenspezifischen Flickenteppich (Braeseke et al. 2019). Obwohl es einen Rechtsanspruch auf Pflegeberatung gemäß $₫ 7$ a SGB XI gibt, kann dieser keineswegs überall in einer den Ansprüchen des Case Managements genügenden Weise eingelöst werden. Das gilt sowohl kassenspezifisch als auch regional. Während die Allgemeinen Ortskrankenkassen (AOK) und wenige Betriebskrankenkassen örtliche und vergleichsweise ausreichende Pflegeberatungsstrukturen vorhalten, gilt dies für viele bundesweit tätige Kassen nicht. Hier finden sich zunehmend Pflegeberatungsangebote, die sich auf eine Hotline konzentrieren und beschränken. Andere Kassen bedienen sich externer Anbieter wie etwa dem Medizinischen Dienst der Krankenkassen (MDK) in Bayern, der eine extra Ausgründung für diese Aufgaben 
vorgenommen hat, oder kommerzieller Case Management-Anbieter, die für mehrere Kassen tätig sind. Ein vergleichsweise suffizientes System haben die privaten Pflegeversicherer mit ihrer Pflegeberatungsfirma compass entwickelt. Diese Lösung erweist sich am ehesten eingebettet in regionale Strukturen und Netzwerke. Auch die Praxis der Pflegestützpunkte unterscheidet sich von Bundesland zu Bundesland und regional erheblich (Braeseke et al. 2019), sowohl was die Konzeption, das Beratungsverständnis und die Kooperationsroutinen als auch die Qualifikation der dort tätigen Fachkräfte anbelangt.

Nur wenige Bundesländer haben eine systematische Verschränkung von Care und Case Management realisiert. Allen voran sind dies das Saarland und Rheinland-Pfalz. Aber auch hier gibt es eine vergleichsweise große Variationsbreite der Case Management-Performance in der Pflegeberatung, je nach Pflegestützpunkt, in den dort in verbindlicher Weise die Pflegeberatung von allen Kassen integriert ist. Sanktionen haben Kassen kaum zu befürchten, wenn sie den Pflegeberatungsanspruch nicht einlösen. Dort, wo es zu Sanktionen kam bei einigen Ersatzkassen und dies nach Prüfung des Bundesverwaltungsamtes -, wurden diese sowohl für die Politik als auch für die Fachöffentlichkeit eher verschwiegen als transparent gemacht. In den Stadtstaaten haben sich zum Teil historisch bedingte Sonderstrukturen herausgebildet, die ebenso die Verbindung zur bezirklichen Altenhilfe kennen wie die Aufnahme bestehender Strukturen, so z. B. im Falle von Berlin mit den Koordinationsstellen für ambulante Rehabilitation. Die Ressourcen für eine aufsuchende Pflegeberatung sind kaum irgendwo in einer Weise vorhanden, die den an sich bestehenden Bedarf befriedigen könnten. Überdies mangelt es regelhaft und auch dort, wo es hinreichend funktionsfähige kommunale Pflegeplanungsstrukturen gibt, an einer systematischen Verschränkung von Case und Care Management mit systematischer Analyse von Infrastrukturdefiziten, Planungsinstrumenten und -verfahren und der Initiative, diese Infrastruktur weiterzuentwickeln. Dabei ist
Case Management nicht nice to have für „leichte und Luxusfälle" im Sinne einer voluntativ und komfortabel in Anspruch genommenen zusätzlichen Beratung. Das Case Management hat, wie beschrieben, auf hochkomplexe Bedarfssituationen $\mathrm{zu}$ reagieren und dies bei besonders vulnerablen Versicherten.

Die Defizite in der Implementierung eines flächendeckenden Care und Case Managements in der Pflege auf der Grundlage von $\$ \$ 7 \mathrm{a}, \mathrm{c}$ SGB XI stehen im deutlichen Widerspruch zu den Erkenntnissen, dass Pflegeberatung für die Betroffenen fühlbare Effekte zeigt. Das konnte schon mit der ersten Evaluation der Pflegeberatung dokumentiert werden (Klie et al. 2011). Auch allgemeine Bevölkerungsbefragungen zur Pflege unterstreichen die fehlende Breitenwirkung der Pflegeberatung. Pflegebedürftige und ihre An- und Zugehörigen nehmen kaum formelle Beratung im Kontext der Pflege in Anspruch. Vielmehr informiert man sich im Bekannten- und Freundeskreis, ggf. über Ärzte. Formelle Beratungsangebote erlangen für die meisten Pflegeerfahrenen in Deutschland bislang keine Bedeutung (Haumann 2018). Die u. a. auch aus den Defiziten in der Implementation der Pflegeberatung entwickelten Vorschläge zu einer Strukturreform Pflege und Teilhabe (Hoberg et al. 2013), die die Verankerung des Care und Case Managements auf der kommunalen Ebene empfahlen, wurden durch die Bund-Länder-Gruppe in der 19. Legislaturperiode in einer Weise verwässert respektive machtpolitisch ausgebremst, dass von der Möglichkeit von Options- respektive Modellkommunen gemäß $\$ \$ 123 f$ SGB XI nur ein einziges Bundesland Gebrauch macht. Es war vorgeschlagen und von 15 Bundesländern und allen drei kommunalen Spitzenverbänden entschieden befürwortet worden, Kommunen die Möglichkeit einzuräumen, gemeinsam mit den Kassen ein integriertes Care und Case Management im Rahmen der Pflegeberatung und der Pflegestützpunkte aufzubauen - und dies mit einer systematisch aufeinander abgestimmten Tätigkeit der beteiligten Akteure und einer einheitlichen Anlaufstelle. $\mathrm{Zu}$ den zentralen Schwachstellen der Pflege- 
beratung in Deutschland gehören ihre fehlende Verfügbarkeit, die faktische Leistungsverweigerung zahlreicher Kassen, die sanktionslos bleibt, die oftmals fehlende Fachlichkeit, unzureichende Ressourcen für die Fallsteuerung, fehlende konsequente Bindung des Case Managements an den Ort und ebenso die nicht konsequente und zum Teil völlig fehlende Verbindung zwischen Case und Care Management (Klie 2020). In der Konsequenz können die eingangs skizzierten Konstellationen, die sich als existentielle Krisen von auf Pflege angewiesenen Menschen darstellen, meist nicht professionell gemanagt werden - mit allen damit verbundenen fachlichen und menschrechtlichen Kollateralschäden.

\subsection{Steuerungsfunktionen des Care und Case Managements}

Die Potenziale einer Case Management-orientierten Arbeitsweise einerseits und einer Case Management-basierten Fallsteuerung im Einzelfall andererseits sind groß, und zwar sowohl im Sinne einer Steigerung der Effizienz des Gesamtsystems als auch im Sinne einer personenzentrierten Lösung von Versorgungs- und Lebenslageproblemen von auf Pflege angewiesenen Menschen. Voraussetzung ist allerdings, dass ein fallbezogenes Case Management sich den oben dargestellten Prinzipien und Qualitätsmerkmalen des Case Managements verpflichtet sieht und die im Einzelfall gewonnenen Erkenntnisse systematisch auf die Systemebene zurückspiegelt, und dies im Sinne eines Care Managements (Monzer 2018).

Die im Einzelfall auftretenden Probleme, die Case Management indizieren, haben höchst unterschiedliche Hintergründe. ${ }^{5}$ Da sind zum

5 Die nachfolgend dargestellten Kategorien und Fallbeispiele entstammen aus Case Management-Implementationsprozessen, die der Autor mit Michael Monzer in Baden-Württemberg, Niedersachsen und im Saarland begleitet. einen personale Probleme, die mit der Person des auf Pflege angewiesenen Menschen verbunden sind. Dabei handelt es sich etwa um psychisch erkrankte Menschen ohne Krankeneinsicht mit Pflegebedarf, die etwa Compliance-Probleme hinsichtlich Hilfen aufweisen. Es können soziale Problemstellungen neben dem pflegerischen Case Management bestehen, etwa wenn in der Familiensituation erhebliche Spannungen bestehen, Menschen in sozialer Isolation leben oder die Ressourcen für die Existenzsicherung nicht ausreichen. Auch Phänomene unzureichender Hygiene, gerne als Vermüllungssyndrom oder Diogenes-Syndrom (Hofmann 1992) bezeichnet, können Case Management indizieren. Diese personalen Probleme lassen sich bei aller Individualität typisieren und es gilt, ggf. Strategien für gleich gelagerte Fälle in Case Management-basierten Vorgehens- und Kooperationsroutinen $\mathrm{zu}$ entwickeln, die wirksame Hilfe in aufeinander abgestimmter Weise gewährleisten. Case Management-Indikationen können sich auch dadurch ergeben, dass die benötigten Hilfen und Dienste zwar vorhanden sind, aber nicht zugänglich gemacht werden, etwa werden MRSA-Patienten aus dem Krankenhaus nicht in eine Kurzzeitpflegeeinrichtung aufgenommen werden, bei sozialhilfeberechtigten Personen Probleme mit der Aufnahme in eine ambulant betreute Wohngemeinschaft bestehen oder Pflegeheime die Aufnahme von BTM-Patienten ablehnen. In diesen Fällen hat das Case Management im Wesentlichen seine Gatekeeper-Funktion wahrzunehmen, im Einzelfall nach Möglichkeit aber auch darüber hinaus die Probleme, die sich als Ablehnung des Systems darstellen, zu überwinden. Case Management-Indikationen können sich überdies aus Versorgungslücken ergeben, sei es, weil für eine bestimmte Zielgruppe keine Angebote zur Verfügung stehen, etwa für junge Menschen mit einem stationären Pflegebedarf oder für Menschen mit SchwerstMehrfach-Behinderung und Pflegebedarf, für die es keine geeigneten oder den Präferenzen entsprechenden Einrichtungen gibt (Klie 2019). Es kann sich allerdings auch um re- 
gionale Versorgungsengpässe handeln, die etwa daraus resultieren, dass ambulante Dienste nicht in der Lage sind, weitere Patienten anzunehmen. Typisch ist auch das Fehlen von solitären Kurzzeitpflegeeinrichtungen, die sowohl im Zusammenhang mit Krisensituationen in der häuslichen Versorgung als auch bei Krankenhausentlassung fehlen. In diesen Konstellationen kommt es in besonderer Weise darauf an, dass die im Einzelfall gesammelten Erfahrungen mit Versorgungsproblemen auf der Systemebene aufgegriffen und im Sinne der Einlösung des Sicherstellungsauftrages für die Weiterentwicklung der Infrastruktur genutzt werden. Versorgungsprobleme, die Case Management indizieren, können auch dergestalt sein, dass zwar Einrichtungsplätze zur Verfügung stehen, die Voraussetzungen für die Aufnahme jedoch nicht gegeben sind - sei es in der Kurzzeitpflege die Verordnung von Medikamenten aus dem Klinikbereich oder fehlende ambulante Palliativangebote jenseits von Fällen der Spezialisierten ambulanten Palliativversorgung (SAPV). Hier kommt dem Case Management die Funktion zu, im Einzelfall die operativen Versorgungsprobleme zu lösen und über den Einzelfall hinaus einen Beitrag dazu zu leisten, damit sich die Prozeduren der Leistungsgewährung respektive der aufeinander abgestimmten Leistungen verbessern lassen. Auch rechtliche Vertretungsmängel bei fehlender Vorsorgevollmacht oder (noch nicht) bestellten gesetzlichen Betreuern können Case Management indizieren, wobei hier streng zu unterscheiden ist zwischen der Notwendigkeit einer sozialen Unterstützung und einer rechtlichen Betreuung im Sinne des $\$ 1896$ BGB. ${ }^{6}$ Gesetzliche Betreuungen sind nicht dazu da, Versorgungsmängel und Defizite in der sozialen Unterstützung zu kompensieren. Schließlich kann Case Management dort indiziert sein, wo die Leistungsgrenzen der jeweiligen Institutionen erreicht sind. Pflegebedürftige mit hirnorganischen Veränderungen (Demenz) können in

6 https://www.justiz.nrw.de/JM/jumiko/beschluesse/ 2018/Fruehjahrskonferenz_2018/I_6.pdf. Zugegriffen: 05. Februar 2020. der eigenen Häuslichkeit besonderen Versorgungsrisiken ausgesetzt sein, die ein ambulanter Dienst oder auch pflegende Angehörige nicht auffangen können, Pflegearrangements mit osteuropäischen Haushaltshilfen können an die Grenzen der fachlichen und kommunikativen Grenzen gelangen, was Versorgungsprobleme und damit Case Management provoziert (AG Garmisch-Partenkirchen, Beschluss vom 28. Mai 2019 - A XVII 9/18 - juris). Kurzzeitpflegeeinrichtungen sind konfrontiert mit Situationen einer nicht sichergestellten Nachversorgung respektive Rückkehr in die eigene Häuslichkeit. Auch hier kann Case Management indiziert sein - und wiederum nicht allein im Sinne der Problemlösung im Einzelfall, sondern (auch) in einer Qualifizierung des Gesamtsystems: Jeder Einzelfall ist ein Anlass zum Lernen für das Gesamtsystem. Eine derartige, konsequent vom Einzelfall her entwickelte Systemsteuerung ist in einem Anbietermarkt, der ganz andere Kalküle für die Angebotsentwicklung kennt als (allein) die örtliche und regionale Bedarfssituation, notwendig, um den Sicherstellungsaufrag im Einzelfall einzulösen und die Vorgaben des Sozialleistungsrechtes umzusetzen, eine bedarfsgerechte wohnortnahe Versorgung zu gewährleisten. Insofern ist ein wirksames Care und Case Management Seismograph für Versorgungsprobleme und Promotor bedarfsgerechter Infrastrukturentwicklung. Eingebettet in regionale Planungsstrategien wird, wie $\bullet$ Abb. 11.1 deutlich macht, das Case Management zu einem zentralen Bestandteil bedarfsgerechter Infrastrukturentwicklung.

Von einem solchen Bild ineinandergreifender Care und Case Management-Strukturen ist die Wirklichkeit der Pflegeberatung und der kommunalen Pflegeplanung weit entfernt (vgl. auch Braeseke et al. 2019). Faktisch bezieht sich die Pflegeberatung, die in den Händen der Pflegekassen liegt, auf die Information über Rechtsansprüche und die Aufstellung eines standardisierten Versorgungsplans. Damit werden mitnichten die mit dem Care und Case Management verbundenen Anliegen umgesetzt und die zum Teil eklatanten Ver- 


\section{Infrastrukturmonitoring und -entwicklung}

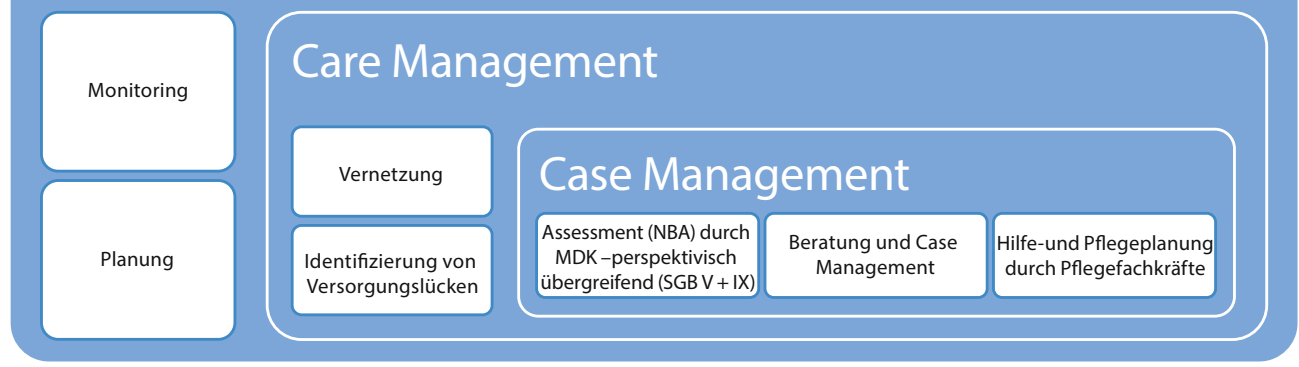

Pflege-Report 2020

- Abb. 11.1 Infrastrukturmonitoring und -entwicklung (Quelle: Klie 2020)

sorgungsprobleme aufgegriffen. Überdies fehlt es an einer umfassenden Awareness-Struktur für Versorgungsprobleme auf Pflege angewiesener Menschen. Diese könnte durch Konzepte wie das der subjektorientierten Qualitätssicherung (Klie und Büscher 2019) mit Hilfe des MDK im Rahmen der Pflegebegutachtung gefördert werden. Eine systematische Nutzung der MDK-Daten aus dem Begutachtungsverfahren könnte zu einer wesentlich größeren Sensitivität des Gesamtsystems gegenüber Problemlagen beitragen und das weithin vernachlässigte Geschehen in der häuslichen Pflege einbeziehen.

\subsection{Ausblick}

Nicht jeder auf Pflege angewiesene Mensch braucht Pflegeberatung und Case Management. Ausgegangen wird von einem Anteil von maximal $5-10 \%$ an den Leistungsbeziehungen der Pflegeversicherung (Klie 2011). Die meisten Versorgungskarrieren werden routiniert im Gesamtsystem gesteuert und mehr oder weniger gut begleitet und umgesetzt. Es gibt allerdings keine Region in Deutschland, die auf Care und Case Management im Sinne einer Case Management-Organisation verzich- ten kann und darf. Zu eklatant sind die Versorgungsprobleme und Infrastrukturdefizite, $\mathrm{zu}$ dramatisch Formen der Unter- und Fehlversorgung von Pflegebedürftigen. Die Pflegepolitik sowohl auf Landes- als auch auf Bundesebene verhält sich dem Care und Case Management gegenüber - mit einer großen Variationsbreite - defensiv. Die rein machtpolitisch motivierte Verhinderung der Verlagerung von Care und Case Management-Kompetenzen auf die kommunale Handlungsebene im Rahmen des Pflegestärkungsgesetzes III ist Ausdruck davon, dass Stakeholder-Interessen relevanter Akteure der gemeinsamen Selbstverwaltung größeren Einfluss haben als zum Teil gravierende Desiderate pflegerischer Versorgung im Einzelfall und vor Ort.

Integrierte Case und Care ManagementStrukturen sind alternativlos, will man eine auf die Effizienz des Gesamtsystems ausgerichtete und zugleich personenzentrierte Fallsteuerung implementieren: Das war die Absicht des Gesetzgebers mit dem Pflege-Weiterentwicklungsgesetz 2008, darauf zielten auch die Vorschläge einer Strukturreform Pflege und Teilhabe 2013 (Hoberg et al. 2013). Die von Ländern, Kommunen und Kassen eingeschlagenen Wege zur Implementierung des Care und Case Managements sind nicht nur höchst variantenreich, sie stehen zum Teil auch in Konkur- 
renz zueinander. Manche Kassen, insbesondere die AOK, investieren in Case ManagementKonzepte, die in eigene regionale Versorgungsstrukturen eingebunden sind, aber nur ihren eigenen Versicherten zur Verfügung stehen. In Selektivverträgen werden regionale Versorgungsnetzwerke gebildet, die wiederum nicht der Bürgerschaft vor Ort insgesamt, sondern nur Teilpopulationen als Adressaten zugutekommt. Manche Bundesländer schaffen integrierte Pflegeberatungsstrukturen in Pflegestützpunkten mit eigenen Finanzmitteln (Saarland, Rheinland-Pfalz), die aber keineswegs immer in dem oben beschriebenen Sinne zu einem integrierten Case und Care Management führen (Braeseke et al. 2019). Andere Kassen wie die DAK investieren in die Entwicklung regionaler Pflegekompetenzzentren, die Case Management-Organisation in virtueller Weise entwickeln - als Akteursnetzwerk mit abgestimmten Vorgehensweisen und gemeinsamer EDV, aber auch Pflegekompetenzzentren als Institutionen, die Infrastrukturdefizite, ggf. gemeinwirtschaftlich, beheben (Klie und Monzer 2018). Aber diese Initiativen werden z.T. als Konkurrenz und nicht als Beitrag zur Sicherung der Daseinsvorsorge in der Langzeitpflege verstanden. So wird man nicht um eine neue bundes- und landesgesetzliche Rahmung herumkommen, die alle Beteiligten zum Aufbau integrierter Versorgungstrukturen verpflichtet und entsprechende Anreize schafft.

\section{Literatur}

Braeseke G, Compagna D, Merda M, Lutze M, Richter T, Weiß C (2013) Abschlussbericht zur Studie „Unterstützung Pflegebedürftiger durch technische Assistenzsysteme". VDI/VDE-IT; Institut für Europäische Gesundheits- und Sozialwirtschaft (IEGUS), Berlin (Hrsg v Bundesministerium für Gesundheit (BMG))

Braeseke G, Pflug C, Beikirch E (2019) Studie zur Erfüllung der Koordinierungs- und Vernetzungsaufgaben sowie der Qualitätssicherung in Pflegestützpunkten. IGES, Berlin

Haumann W (2018) Bilder und Erfahrungen der Pflege in Deutschland und in den Bundesländern. In: Klie
T (Hrsg) Pflegereport 2018. Pflege vor Ort - gelingendes Leben mit Pflegebedürftigkeit. Beiträge zur Gesundheitsökonomie und Versorgungsforschung, Bd. 26. medhochzwei, Heidelberg, S 31-108 (Hrsg von Storm A und DAK-Gesundheit)

Hoberg R, Klie T, Künzel G (2013) Strukturreform Pflege und Teilhabe. Langfassung. FEL Verlag Forschung Entwicklung - Lehre, Freiburg

Hofmann W (1992) Das Diogenes-Syndrom: Leben zwischen allerlei Krimskrams. In: Geriatrie-Praxis. MMVMedizinverlag, München

Klie T (2011) Pflegeversicherung erklärt von Thomas Klie. Dr. med. Mabuse 36(193):42

Klie T (2014) Wider den „Pflegefall“ und andere sprachliche Katastrophen! Demenz. Leben 20:22-23

Klie T (2018) Der DAK-Pflegereport 2018: Gleichwertige Lebensbedingungen für die Pflege? Zentrale Erträge und Denkanstöße. In: Klie T (Hrsg) Pflegereport 2018. Pflege vor Ort - gelingendes Leben mit Pflegebedürftigkeit. Beiträge zur Gesundheitsökonomie und Versorgungsforschung, Bd. 26. medhochzwei, Heidelberg, S 3-30 (Hrsg von Storm A und DAKGesundheit)

Klie T (2019) Pflegereport 2019. 25 Jahre Pflegeversicherung: Kosten der Pflege - Bilanz und Reformbedarf. Beiträge zur Gesundheitsökonomie und Versorgungsforschung, Bd. 30. medhochzwei, Heidelberg (Unter Mitarbeit von Haumann W, Hildebrandt $\mathrm{H}$, Lange L, Lewin P, Moeller-Bruker C, Pfeil J et al. Hrsg von Storm $A$ und DAK-Gesundheit)

Klie T (2020) Pflegeberatung und Pflegestützpunkte zwischen pflegepolitischer Bedeutung und Wirklichkeit. In: Löcherbach P, Wendt WR (Hrsg) Care und Case Management, 1. Aufl. Kohlhammer, Stuttgart

Klie T, Büscher A (2019) Subjektorientierte Qualitätssicherung in der Langzeitpflege. Nachrichtend Dtsch Ver 99(3):114-119

Klie T, Monzer M (2018) Regionale Pflegekompetenzzentren. Innovationsstrategien für die Langzeitpflege vor Ort. Beiträge zur Gesundheitsökonomie und Versorgungsforschung, Bd. 25. medhochzwei, Heidelberg

Klie T, Frommelt M, Schneekloth U, Behrend S, Göhner A, Heislbetz C et al (2011) Evaluation der Pflegeberatung nach $\S 7 \mathrm{a}$ Abs. 7 Satz 1 SGB XI. Bundesweiter Überblick Nr. 1-6c. GKV-Spitzenverband

Klie T, Krahmer U, Plantholz M (Hrsg) (2014) Sozialgesetzbuch XI - Soziale Pflegeversicherung, 4. Aufl. Lehrund Praxiskommentar (LPK-SGB XI). Nomos, BadenBaden

Koch-Straube U (2008) Beratung in der Pflege. Huber, Bern

Monzer M (2018) Case management organisation, 1. Aufl. medhochzwei, Heidelberg

Petermann A, Ebbing T, Paul M (2017) Das Tätigkeitsprofil von Betreuungspersonen in häuslicher Gemein- 
schaft. Berufsakademie für Gesundheits- und Sozialwesen Saarland, Saarbrücken

Rothgang H, Müller R (2019) Pflegereport 2019 -

Ambulantisierung der Pflege. BARMER (Hrsg)

Schriftenreihe zur Gesundheitsanalyse, Band 20.

Berlin. https://www.barmer.de/blob/215396/ a68d16384f26a09f598f05c9be4ca76a/data/dlbarmer-pflegereport-2019.pdf. Zugegriffen: 20. Febr. 2020

Open Access Dieses Kapitel wird unter der Creative Commons Namensnennung 4.0 International Lizenz (http:// creativecommons.org/licenses/by/4.0/deed.de) veröffentlicht, welche die Nutzung, Vervielfältigung, Bearbeitung, Verbreitung und Wiedergabe in jeglichem Medium und Format erlaubt, sofern Sie den/die ursprünglichen Autor(en) und die Quelle ordnungsgemäß nennen, einen Link zur Creative Commons Lizenz beifügen und angeben, ob Änderungen vorgenommen wurden.

Die in diesem Kapitel enthaltenen Bilder und sonstiges Drittmaterial unterliegen ebenfalls der genannten Creative Commons Lizenz, sofern sich aus der Abbildungslegende nichts anderes ergibt. Sofern das betreffende Material nicht unter der genannten Creative Commons Lizenz steht und die betreffende Handlung nicht nach gesetzlichen Vorschriften erlaubt ist, ist für die oben aufgeführten Weiterverwendungen des Materials die Einwilligung des jeweiligen Rechteinhabers einzuholen. 